\title{
Juvenile Crime Monitoring and Characteristic Analysis Based on the Internet of Things and Grid Management
}

\author{
Jingyi Gong \\ Law School, Sichuan Agricultural University, Ya'an, Sichuan Province 625014, China \\ Correspondence should be addressed to Jingyi Gong; 201901830@stu.sicau.edu.cn
}

Received 26 November 2021; Revised 17 December 2021; Accepted 23 December 2021; Published 11 February 2022

Academic Editor: Hasan Ali Khattak

Copyright ( 2022 Jingyi Gong. This is an open access article distributed under the Creative Commons Attribution License, which permits unrestricted use, distribution, and reproduction in any medium, provided the original work is properly cited.

\begin{abstract}
Over the past decade, researchers have identified intervention methods and program models that reduce juvenile delinquency and encourage prosocial development. Preventing juvenile delinquency not only saves young lives from being wasted but also prevents the onset of adult criminal careers and thus reduces the burden of crime on its victims and society. Aiming at this social problem, this study combines Internet of Things and grid models to study the attribution and prevention mechanisms of minor campus violence crimes. It realizes the modern network system mode of information management, collaborative operation, and grid integration in the target area through Internet of Things and grid models. In addition, based on consulting-related literature and practical research, the causes of campus violence crimes are analyzed through a self-organizing competitive neural network. Finally, this article combines the judicial practice of the management of campus violence and presents the prevention and control countermeasures of juvenile campus violence crimes to effectively prevent and control the occurrence of juvenile campus violence crimes.
\end{abstract}

\section{Introduction}

In recent years, school violence, especially juvenile school violence crimes, has become increasingly prominent. It has become a hot issue of public concern and should arouse high vigilance from all walks of life [1]. The soft children's minds can be impressed and curved towards crimes by various elements such as family background, education, financial problems, easy online video accessibility, Internet, friend groups, psychological issues, drugs, and media [2]. Family is the erudition center for children to acquire good or bad qualities. The rejected children by family or children living with guardians or homeless children are found at a high risk of becoming criminals. In developing the personality of children, school education also plays a vital role. Certain crucial elements in the schooling setup such as failures in examinations, expulsions, punishments, and school dropout also increase juvenile offense cases [3].

The Internet has brought unprecedented changes to our lives, and it has also brought a huge impact on people's thinking, especially youths [4]. Because the mental and physical development of youths is not sound, their discrimination ability is limited, and they are not enough to cope with the different types of information on the Internet. They are often lost in the online Internet world. The nature of pursuing novel things makes them constantly explore this unknown realm to indulge in it [5]. Moreover, the bad information on the Internet, such as violent and bloody games, websites that create fear as fun, and various pornographic contents, are constantly distorting the simple hearts of minors [6]. For this reason, many minors have embarked on the path of crime [7]. School violence is a kind of violence which is easy to accept and understand literally, but there is no clear consensus on the definition of violence [8]. There are currently two main points of view. One is the definition of Olworth [9], which believes that violence is an act of assault, in which an actor uses his own body or an object (including weapons) to cause (more serious) harm to others. The other is the definition of the World Health Organization, which believes that violence is the deliberate use of physical power or rights to cause or be likely to cause mental harm, damage, death, deformity, or deprivation of 
benefits to oneself, others, or society" [10]. Criminal psychologists generally define violence as an "aggressive behavior" that intends to cause physical or psychological harm to others. Based on the opinions of domestic and foreign scholars [11], the authors believe that violence includes both physical violence (such as physical attacks or aggressive behavior with the help of tools), psychological violence (such as insults, intimidation, and insults), and verbal violence.

Numerous classification and clustering systems available in data mining were used for juvenile crime identification, detection, and analysis. Ates et al. [12] provided a summary of the use of data mining and machine learning techniques in crimes and gave a new perception of the decision-making processes by presenting examples of the use of data mining for a crime. Saroja Thota et al. [13] extended the work of association rule mining of juvenile delinquency with two key risk elements, family background and education levels of children involved in crimes. They also discovered the rules connecting family background, education levels, and juvenile delinquency with the Indian juvenile crime dataset by association rule mining, a rule-based machine learning mechanism. Takahashi and Evans [14] examined the impact of the individual and social background in forecasting the rearrest of juveniles who were brought into the juvenile crime detention center. Demographic characteristics such as first offense type, gang association, drug usage, and family characteristics were investigated, using cross validation and a machine learning model. Josja Rokven et al. [15] examined the differences and similarities between juvenile delinquents of self-reported cyber-enabled offenses, cyber-dependent offenses, and offline offenses. The online and offline offenses among juveniles aged $12-17$ years old were evaluated. It was reported that juveniles who committed both offline and online crimes were at the high-risk profile. Within the group of online delinquents, juveniles who committed both cyberdependent and cyber-enabled offenses have the highest risk profile. It was determined that cyber-dependent delinquents are a distinct group from online delinquents. The authors in [16] reported that online criminals were often prone to offline crime as well. These results were based on a sample of undergraduate college students. However, the question is whether their findings can be applied to a wider population of juveniles. Bagaric et al. [17] examined the application of algorithms to predict future offenses of juvenile and analyzed the inborn resistance that juvenile has towards deferring decisions.

In this study, the basic definition of juvenile violent crimes on campus is carried out. The characteristics of juvenile violent crimes on campus are examined, and empirical research is conducted to analyze the current trend of juvenile campus violence crime. The collected juvenile campus violence crime cases are classified, and the current development trend of juvenile campus violence crime is analyzed. In addition, the reasons for the insufficient prevention of minor campus violence crimes are investigated by combining Internet of Things (IoT) and the grid management model. Through the investigation of juvenile campus violence crimes and specific cases, the current prevention of juvenile campus violence crimes is analyzed from five aspects: legal system level, school education level, social management level, family education level, and psychological intervention level.

The rest of the paper is organized as follows: Section 2 provides an overview of juvenile delinquency. Section 3 illustrates the proposed crime management method based on Internet of things and grid models. Section 4 provides a case study to illustrate the results of the proposed model, and Section 5 is about the conclusion.

\section{Overview of Juvenile Delinquency}

Juvenile delinquency is the criminal and antisocial actions committed by an individual under the age of 18 . Once a person reaches adulthood, then his antisocial and illegal behavior is known as crime. In this sense, juvenile delinquency is the child and adolescent version of crime [18]. Juvenile delinquency includes two general forms of behaviors, delinquent offense and status offense. Status offenses are behaviors that are considered unsuitable or unhealthy for adolescents and juveniles, and the behaviors are forbidden because of the age of the criminal.

In recent years, the problem of juvenile delinquency has been particularly prominent in countries around the world, and it has attracted great attention from all nations all over the world. Sociology experts, teachers, and other people from all walks of life who are concerned about juvenile delinquency have formulated many constructive methods, but they still cannot effectively control the rise in juvenile delinquency [18].

Juvenile delinquency is significant in society for several reasons, especially for three reasons. First, juveniles and teenagers commit a significant amount of crimes that result in violence, property, or other kinds of victimization. Every year, more than one million adolescents and children are arrested by police for their antisocial acts. Second, juvenile delinquency is itself realized as a pointer for the general health of a society. In zones with high levels of delinquency, antisocial behavior is seen as part of a larger set of community problems. In this sense, juvenile delinquency is worrying because of the victimizations that are inflicted and the perceptual image of society as unable to adequately control and supervise young people. Third, juvenile delinquency has a variety of definitions depending on different factors and its severity. For many young individuals, juvenile delinquency is a normal pace of growing up. For a small class of youths, however, juvenile delinquency is simply the initial stage of what will become a lifetime of antisocial behavior [19].

The prevention of juvenile delinquency can be divided into a narrow sense and a broad sense. In a narrow sense, it refers to the prevention of juvenile crimes, that is, research to prevent and eliminate the causes of crimes that may or have begun to form on them, to prevent or eliminate the crime conditions that may occur around them, so that they do not commit crimes as much as possible [20]. In a broad sense, the prevention of juvenile crimes includes adolescents who have not committed crimes, preventing them from committing crimes, and also includes minor violations of the law 
and adolescents with delinquent deeds, preventing them from committing serious crimes. Figure 1 shows the trend and main scope of juvenile delinquency.

Through the investigation of these cases, it is found that this is closely related to the minor family environment [21]. Juveniles at a young age are in a state of reluctance to understand their physical and mental development, lack of social experience, insufficient knowledge of things, and extremely weak legal awareness. They are in a period of development of world outlook, outlook on life, and values. They commit crimes due to the lack of enough care by family, school, society, and proper education.

\section{Crime Management Based on Internet of Things and Grid Models}

Aiming at the problem of juvenile crimes, this article combines IoT and the grid model to investigate the attribution and prevention mechanism of juvenile campus violence crimes. This study realizes the modern network system model of information management, collaborative operation, and grid integration in the target area through IoT and the grid model. In addition, based on consultingrelated literature and practical research, the causes of campus violence crimes are analyzed through a self-organizing competitive neural network.

\subsection{Juvenile Crime Management Based on the Grid Model.} A grid model is a well-organized model with a set of knowledge management operations. A well-organized model guarantees knowledge to be accurately identified. This study employed grid management to solve the problem of juvenile crime management. Grid management is an emerging management method under the modern network system model of information management, collaborative operation, and grid integration based on the idea of gridding in the target area [22]. The advantages of grid management are reflected in the system structure with the characteristics of "grid layout, integration of blocks," resources with the characteristics of "resource sharing, paid to finance," information with the characteristics of "information integration and degree of access," and business processes with "operation coordination and orderly precession" $[23,24]$. The basic unit of the grid management model is perfect, and the layout is decentralized. A rectangular grid or circular grid can be selected. Figure 2 shows a schematic diagram of the grid management model.

Assuming that the basic unit of the grid management model can be represented by $y_{i}(i=1,2, \ldots, n)$, its vector form is

$$
y=\left[y_{1}, y_{2}, \ldots, y_{n}\right]^{T} .
$$

The form of the set is

$$
Y=\left\{y_{1}, y_{2}, \ldots, y_{n}\right\} .
$$

The management of juvenile crimes adopts a packaged treatment method, divides the grid, and uses the general equation of state to express, which is called the process equation.

$$
x(t+1)=H^{T}(t) x(t),
$$

where $x(t)$ represents the task flow vector at time $t, x(t)=$ $\left[x_{1}(t), x_{2}(t), \ldots, x_{n}(t)\right]^{T}$ is set, and $H(t)$ shows the dynamic relationship matrix corresponding to $x(t) . H(t)$ is the actual operation; the operation is selected to a certain extent to change the dynamic structure of the system. To adapt to this situation, a "decision variable" $d_{\mathrm{ij}}(t)$ is introduced.

$$
H(t)=D(t) \otimes K,
$$

where $K$ is the basic incidence matrix and the correlation coefficient is $K_{\mathrm{ij}}$. $\mathrm{K}=\left[K_{\mathrm{ij}}\right]$, and $K_{\mathrm{ij}}$ is the correlation coefficient of the grid unit. When the value of the correlation coefficient is 0 , it shows that there is no correlation. When the value is 1 , it means there is a correlation. The value is a real number from 0 to 1 and represents the strength of its association. $D(t)$ is the decision matrix, and the decision coefficient is $d_{\mathrm{ij}}(t)$.

For the dynamic incidence matrix $H(t)$, the dynamic correlation coefficient is $h_{\mathrm{ij}}(t)$, and the definition is computed as

$$
h_{\mathrm{ij}}(t)=d_{\mathrm{ij}}(t) \times k_{\mathrm{ij}} .
$$

The dynamic correlation coefficient $h_{\mathrm{ij}}(t)$ has the following characteristics:

$$
\left\{\begin{array}{l}
0 \leq h_{\mathrm{ij}}(t) \leq 1, \\
0 \leq \sum_{j=1}^{n} h_{\mathrm{ij}}(t) \leq 1 .
\end{array}\right.
$$

Through the above constraints, "decision" and "control" can only be selected under the constraints [24]. Using the mathematical expression of the grid management process, the task management is quantitatively calculated to complete the unification of the task points within a given range and a certain distance from the grid, and realizes the transformation from qualitative analysis to quantitative analysis. It is also a grid management model.

3.2. Analysis of Crime Characteristics Based on the Self-Organizing Competitive Neural Network. The self-organizing competitive neural network model performs self-organization and self-judgment on the input and finally divides our input into different types [25]. This study evaluates the implementation effect according to the effect of juvenile crime management. The competitive network is divided into the competition layer and the input layer. Figure 3 shows the model structure of the self-organizing competitive neural network.

The self-organizing competitive neural network can not only be used to study the topological structure of the input sample and the distribution of sample features but also can identify the area near the input sample [26]. The initial weights of the neural network generally use relatively small randomly generated constants so that the weight vector can 


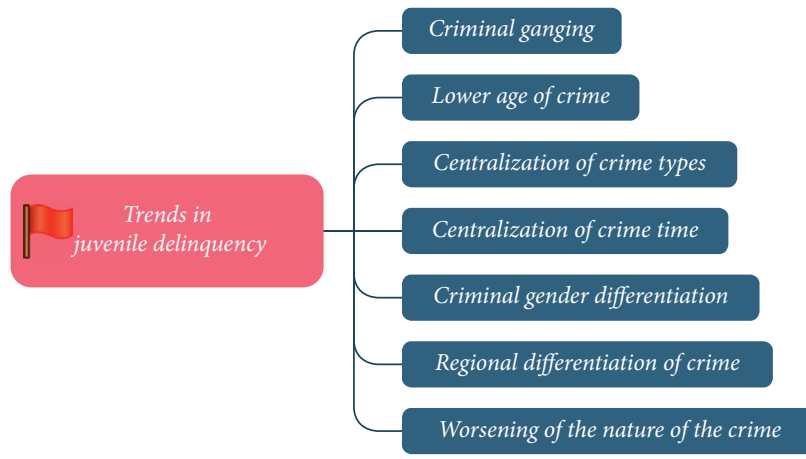

Figure 1: Trends and the main scope of juvenile delinquency.
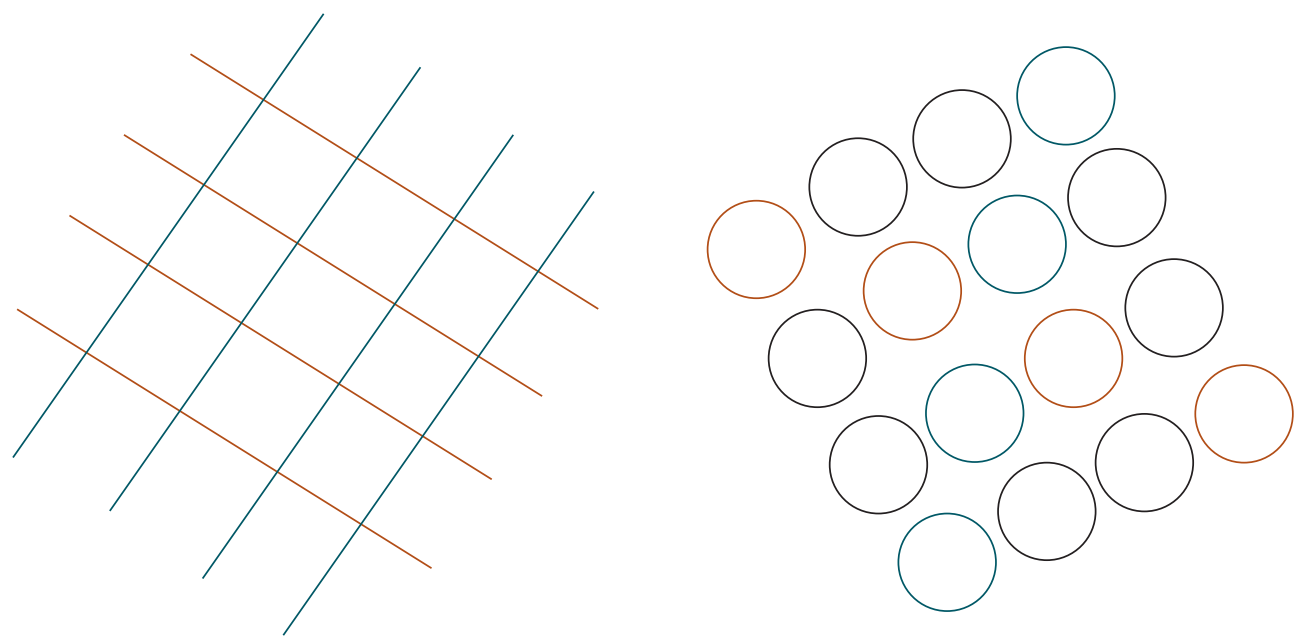

Figure 2: Schematic diagram of the grid management model.

be more fully distributed in the sample space. But in practice, some samples are often concentrated in certain areas of the space, while the initial weight vector is distributed in the entire sample space [27]. The competition layer is composed of ' $m$ ' neurons, and the input layer is composed of ' $n$ ' neurons.

$$
\begin{aligned}
\sum_{i=1}^{n} A_{\mathrm{ij}} & =1, \\
u_{j} & =\sum_{i=1}^{n} w_{\mathrm{ij}} x_{i}-\theta_{j} .
\end{aligned}
$$

In the competition layer, neurons compete with each other. To adapt to the input sample, only one or a few neurons win. The winning neuron or neurons represents the classification model of the given sample.

$$
h_{j}=f\left(u_{j}\right)=\frac{1}{1-\exp \left(-u_{j}\right)} .
$$

The number of neurons in the output layer is important for dividing the data $[28,29]$. If the scale of the network is too small and the data are too dense, it will not be possible to find many differences between the data that should be paid attention to [30]. On the contrary, if the scale of the network is too large, the data that should be brought together will be scattered. This study assumes that the number of nodes in the output layer of the neural network should be appropriate in line with the heuristic network law used to find diatoms.

$$
l_{t}=\sum v_{\mathrm{jt}} h_{j}-\gamma_{t}
$$

This study selected part of the juvenile crime management tasks to participate in the competition data, and the rest are used as samples for this question test. We divided the implementation effect into three grades, namely, the excellent implementation effect, the medium implementation effect, and the poor implementation effect.

$$
\begin{aligned}
y_{t} & =\frac{1}{1+\exp \left(l_{t}\right)}, \\
\varepsilon_{i} & =\left(c_{t}-y_{t}\right) y_{t}\left(1-y_{t}\right) .
\end{aligned}
$$

Therefore, we set 3 neurons. To make the evaluation more accurate, we set the error to 0.1 . Then, the normalized data defined $x^{\prime}$ is the original data, and $x_{\max }$ is the maximum value of the original data and defined $x_{\min }$ as the minimum value of the original data. 


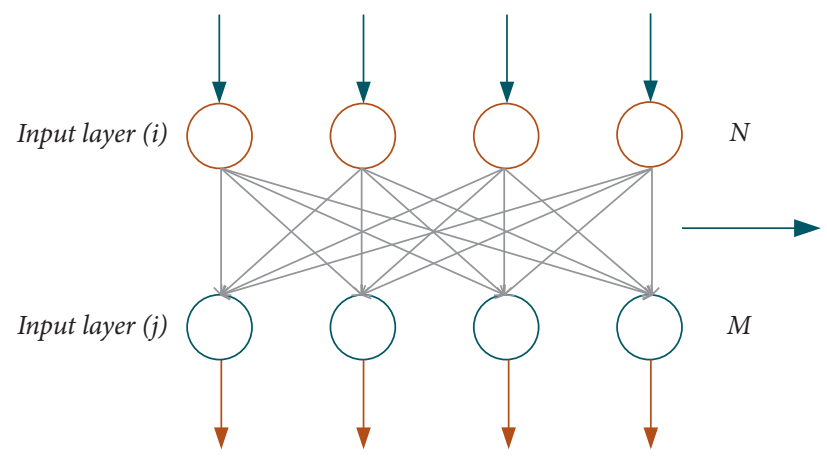

FIGURE 3: Model structure of the self-organizing competitive neural network.

$$
\varepsilon_{j}=\sum_{i=1}^{q} \varepsilon_{i} v_{\mathrm{jt}} h_{j}\left(1-h_{j}\right)
$$

Therefore, we can compress the original data, and the compression interval is $[0,1]$, which plays a certain role in the training of the neural network.

$$
x=\frac{x^{\prime}-x_{\min }^{\prime}}{x_{\max }^{\prime}-x_{\min }^{\prime}} .
$$

\section{Case Analysis of Juvenile Crimes}

This study takes N City as an example. A total of 160 cases of minor campus violence crimes occurred in N City (including the cities and counties under its jurisdiction) from 2017 to 2020 , including 36 cases of quarreling and provocation and 54 cases of gatherings to fight. There were 47 intentional injuries, 13 robberies, 6 rapes, and 4 intentional homicides. This study analyzed the cases of minor campus violence crimes accepted by the public security organs of N City and examined the current situation of minor campus violence crimes in this city. The ages of the minors were between 14 and 18 years. The scope of the campus was mainly defined as the reasonable radiation scope of primary and secondary school campuses, vocational schools, and their surroundings. In a broad sense, the prevention of juvenile crimes includes minors who have not committed crimes, preventing them from committing crimes, and also includes minor violations of the law and minors with delinquent deeds, preventing them from committing serious crimes. The statistical types of crimes mainly include violent crimes such as provoking troubles, gathering people to fight, intentionally hurting, intentionally killing, robbery, and rape. Through the investigation of juvenile campus violence crimes and specific cases, it analyzes the current prevention of juvenile campus violence crimes from five aspects: legal system level, school education level, social management level, family education level, and psychological intervention level. Table 1 describes the latitude, longitude, and evaluation status of juvenile crime cases.

The task numbers corresponding to the numerical division of the self-organizing competitive neural network and the corresponding actual categories are shown in Table 2, where 1 represents that the implementation effect is
TABle 1: The latitude, longitude, and assessment of juvenile crime cases.

\begin{tabular}{lccc}
\hline $\begin{array}{l}\text { Case } \\
\text { number }\end{array}$ & $\begin{array}{c}\text { Case GPS } \\
\text { latitude }\end{array}$ & $\begin{array}{c}\text { Case } \\
\text { number }\end{array}$ & $\begin{array}{c}\text { Case GPS } \\
\text { longitude }\end{array}$ \\
\hline C001 & 22.65 & 114.34 & 76.87 \\
C002 & 22.71 & 114.24 & 72.91 \\
C003 & 22.72 & 114.29 & 75.65 \\
C004 & 22.74 & 114.28 & 75.59 \\
C0015 & 22.73 & 114.29 & 75.75 \\
$\ldots \ldots$ & & & \\
C156 & 23.20 & 113.33 & 54.17 \\
C157 & 23.20 & 113.33 & 53.89 \\
C158 & 23.19 & 113.26 & 48.01 \\
C159 & 23.22 & 113.27 & 49.49 \\
C160 & 23.15 & 113.36 & 54.97 \\
\hline
\end{tabular}

excellent, 2 indicates that the implementation effect is medium, and 3 represents that the implementation effect is poor. Through the mathematical expression of the grid management process, the task management is quantitatively calculated to complete the unification of the task points within a given range and a certain distance from the grid, and realized the transformation from qualitative analysis to quantitative analysis. It is also a grid management model. Table 2 describes the evaluation table of the implementation effect of juvenile crime through grid management.

It can be seen from Table 2 that the self-organizing neural network model evaluates the proportion of "excellent implementation effect" and "medium implementation effect," which is much higher than that of "poor implementation effect," indicating that the management plan given in this study is feasible for the project. The problem of juvenile delinquency caused by the Internet is a complex social problem. The Internet is a direct cause, but it is not only caused by the emergence of the Internet but is the result of various negative factors in society acting on minors. It has the characteristics of sociality and comprehensiveness. Therefore, it is impossible to solve this problem by a single department or a certain method. Instead, it must rely on the power of the whole society to work together and manage it through political, economic, administrative, legal, and cultural issues. Juvenile delinquency can be prevented by implementing a complete school-wide approach that includes guaranteeing that the school has supportive managerial leadership, providing high-grade need-based 
TABLE 2: Output values of the self-organizing competitive neural network.

\begin{tabular}{|c|c|c|c|c|c|c|c|}
\hline Case number & Value & Case number & Value & Case number & Value & Case number & Value \\
\hline $\mathrm{C} 001$ & 2 & $\mathrm{C} 017$ & 3 & C56 & 2 & C149 & 1 \\
\hline C002 & 2 & C018 & 1 & C57 & 2 & $\mathrm{C} 150$ & 3 \\
\hline $\mathrm{C} 003$ & 2 & C019 & 1 & C58 & 2 & $\mathrm{C} 151$ & 3 \\
\hline C004 & 2 & $\mathrm{C} 020$ & 1 & C59 & 2 & $\mathrm{C} 152$ & 3 \\
\hline C005 & 2 & $\mathrm{C} 021$ & 1 & C60 & 2 & C153 & 1 \\
\hline C006 & 2 & $\mathrm{C} 022$ & 1 & C61 & 2 & C154 & 3 \\
\hline $\mathrm{C} 007$ & 2 & $\mathrm{C} 023$ & 1 & C62 & 2 & C155 & 3 \\
\hline$\ldots \ldots$ & & & & & & & \\
\hline C012 & 1 & C58 & 2 & $\mathrm{C} 144$ & 1 & C162 & 3 \\
\hline $\mathrm{C} 013$ & 1 & C59 & 2 & C145 & 3 & C163 & 3 \\
\hline C014 & 1 & C53 & 2 & C146 & 3 & C164 & 1 \\
\hline C015 & 1 & C54 & 2 & $\mathrm{C} 147$ & 3 & C165 & 1 \\
\hline C016 & 1 & C55 & 2 & C148 & 3 & $\mathrm{C} 160$ & 3 \\
\hline
\end{tabular}

professionals, using academic and behavioral scrutiny for the identification of at-risk youth, integrating programs, and implementing continuous program evaluation and modification. At-risk students must be identified early, and student activities must be monitored and tracked regularly. In this way, incidents of juvenile delinquency may be significantly reduced.

\section{Conclusion}

Recently, juvenile school violence crimes have become increasingly prominent and have become an important issue of public concern. This study analyzed the current status, characteristics, and various types of behavior of minors due to cybercrimes and discussed the various reasons for this problem from the family, school, community, and society. To reduce the number of minors committing crimes, it is proposed to establish a monitoring system in which the whole society should be collectively managed and corrected the deviations of criminal minors to prevent them from committing crimes again. This study employed the modern network system model of information management, collaborative operation, and grid management in the target area through IoT and the grid model. In addition, based on consulting-related literature and practical research, the cause of campus violence crimes is analyzed through a selforganizing competitive neural network. It draws on a lot of advanced experience and puts forward some new insights on improving the effective supervision of network information and perfecting administrative legislation. Finally, the prevention and control countermeasures of juvenile campus violence crimes are presented to effectively prevent and control the occurrence of juvenile campus violence crimes.

\section{Data Availability}

The data used to support the findings of this study are included within the article.

\section{Conflicts of Interest}

The author declares that there are no conflicts of interest.

\section{References}

[1] A. Mimi, "crime, social control, and the process of social classification: juvenile delinquency/justice discourse in Israel," Social Problems, vol. 4, no. 4, pp. 1948-1970, 2014.

[2] P. Liana, "A case study approach to procedural justice: parents' views in two juvenile delinquency courts in the United States," British Journal of Criminology, vol. 55, no. 5, p. 901, 2015.

[3] G. W. J. M. Stevens, V. C. Veen, and W. A. M. Vollebergh, "Psychological acculturation and juvenile delinquency: comparing Moroccan immigrant families from a general and pretrial detention population," Cultural Diversity and Ethnic Minority Psychology, vol. 20, no. 2, pp. 254-265, 2014 Apr.

[4] V. Hester, "Eeren, "value of information analysis applied to the economic evaluation of interventions aimed at reducing juvenile delinquency: an illustration," PLOS ONE, vol. 10, no. 7, pp. 154-162, 2015.

[5] A. J. Shelley and A. J. Shelley, "Biossistemática e distribuição de simulídeos vetores da oncocercose humana na América do Sul," Early Intervention in Psychiatry, vol. 8, no. 1, pp. 87-90, 2014.

[6] Elizabeth Moore, Devon Indig, and Leigh Haysom, "Traumatic brain injury, mental health, substance use, and offending among incarcerated young people," The Journal of Head Trauma Rehabilitation, vol. 29, no. 3, pp. 239-247, 2014.

[7] J. B. Folk, A. Harrison, and C. Rodriguez, "Feasibility of social media-based recruitment and perceived acceptability of digital health interventions for caregivers of justice-involved youth: mixed methods study," Journal of Medical Internet Research, vol. 22, no. 4, 2020.

[8] M. J. Brooks, K. Abebe, E. Miller, and E. P. Mulvey, "The longitudinal relationship between future orientation and substance use among youth with serious criminal offenses," Journal of Adolescent Health, vol. 60, no. 2, p. S11, 2017.

[9] S. Subramanian, "' Socio-demographic characteristics and aggression quotient among children in conflict with the law in India: a case-control study," The National medical journal of India, vol. 28, no. 4, pp. 172-175, 2015.

[10] K. W. Nilsson, C. Erika, and H. Sheilagh, "Genotypes do not confer risk for delinquency ut rather alter susceptibility to positive and negative environmental factors: gene-environment interactions of BDNF Val66Met, 5-HTTLPR, and MAOA-uvntr," International Journal of Neuropsychopharmacology, vol. 18, no. 5, p. 5, 2015.

[11] E. Moore, D. Indig, and L. Haysom, "Traumatic brain injury, mental health, substance use, and offending among 
incarcerated young people," The Journal of Head Trauma Rehabilitation, vol. 29, no. 3, pp. 239-247, 2014.

[12] E. C. Ates, E. Bostanci, and M. S. Guzel, "Big data, data mining, machine learning, and deep learning concepts in crime data' (2020) 8(2) ceza hukuku ve kriminoloji dergisi," Journal of Penal Law and Criminology, vol. 293, 2020.

[13] L. Saroja Thota, K. Shireesha, A. Sravani, and S. Rajender, "Rule-based mining of juvenile delinquency," in Proceedings of the 2020 International Conference on Computer Communication and Informatics (ICCCI -2020), IEEE, Coimbatore, INDIA, 22 January 2020.

[14] Y. Takahashi and L. T. Evans, "An application of machine learning for predicting rearrests: significant predictors for juveniles," Race and Social Problems, vol. 10, no. 1, pp. 42-52, 2018.

[15] J. Josja Rokven, G. Weijters, G. C. Marinus Beerthuizen, and M. André, "Juvenile delinquency in the virtual world: similarities and differences between CyberEnabled, cyber-dependent and offline delinquents in The Netherlands," International Journal of Cyber Criminology, vol. 12, no. 1, 2018.

[16] C. M. Donner, W. G. Jennings, and J. Banfield, "The general nature of online and off-line offending among college students," Social Science Computer Review, vol. 33, no. 6, pp. 663-679, 2015.

[17] M. Bagaric, D. Hunter, and N. Stobbs, "Erasing the bias against using artificial intelligence to predict future criminality: algorithms are color blind and never tire," University of Cincinnati Law Review, vol. 88, no. 4, Article ID 31037-1081, 2019.

[18] C. V., C. J. . Andrea, "Social use of alcohol among adolescent offenders: a fundamental approach toward human needs," Revista da Escola de Enfermagem da USP, vol. 48, no. 1, pp. 135-143, 2014.

[19] K. A. Meerja, P. V. Naidu, and S. Kalva, "Price versus performance of big data analysis for cloud-based internet of things networks," Mobile Networks and Applications, vol. 24, no. 9, pp. 1-17, 2019.

[20] M. Noura, M. Atiquzzaman, and M. Gaedke, "Interoperability in internet of things: taxonomies and open challenges," Mobile Networks and Applications, vol. 24, no. 7, pp. 1-14, 2019.

[21] J. Iannacci, G. Resta, and A. Bagolini, "RF-MEMS monolithic $\mathrm{K}$ and ka-band multi-state phase shifters as building blocks for 5G and internet of things (IoT) applications," Sensors, vol. 20, no. 9, 2020.

[22] A. Aslam, U. Mehmood, M. H. Arshad et al., "Dye-sensitized solar cells (DSSCs) as a potential photovoltaic technology for the self-powered internet of things (IoTs) applications," Solar Energy, vol. 207, pp. 874-892, 2020.

[23] N. Pearre and L. Swan, "Reimagining renewable electricity grid management with dispatchable generation to stabilize energy storage," Energy, vol. 203, 2020.

[24] X. Fan, Y. Li, and L. Luo, "Groundwater pollution detection and power grid enterprise human resource management based on Markov chain," Arabian Journal of Geosciences, vol. 14, no. 15, pp. 1-16, 2021.

[25] J. R. Kala, D. M. Kre, and A. N. Gnassou, "Assets management on an electrical grid using Faster-RCNN[J]," Annals of Operations Research, vol. 308, no. 1, 2020.

[26] M. Shakir and Y. Biletskiy, "Forecasting and optimization for microgrid in-home energy management systems," IET Generation, Transmission \& Distribution, vol. 14, no. 17, 2020.

[27] C. Guzman, A. Cardenas A, and K. Agbossou, "Local estimation of critical and off-peak periods for grid-friendly flexible load management," IEEE Systems Journal, vol. 14, no. 99, pp. 1-10, 2020.

[28] H. Liang, "Evaluation of fitness state of sports training based on self-organizing neural network," Neural Computing \& Applications, vol. 33, no. 9, pp. 3953-3965, 2021.

[29] K. Huang, X. Ma, R. Song, X. Rong, and Y. Li, "Autonomous cognition development with lifelong learning: a self-organizing and reflecting cognitive network," Neurocomputing, vol. 421, pp. 66-83, 2021.

[30] M. Music, G. Parigi, and V. Cantoni, “A scalable multi-signal approach for the parallelization of self-organizing neural networks," Neural Networks, vol. 123, pp. 108-117, 2020. 